\title{
NONLINEAR ANALYSIS OF STABILITY FOR IMPERFECT ECCENTRICALLY STIFFENED FGM PLATES UNDER MECHANICAL AND THERMAL LOADS BASED ON FSDT. PART 1: GOVERNING EQUATIONS ESTABLISHMENT
}

\author{
Dao Van Dung, Nguyen Thi Nga* \\ VNU University of Science, Hanoi, Vietnam \\ *E-mail: nguyennga282@gmail.com \\ Received February 02, 2015
}

\begin{abstract}
In this paper, the buckling and post-buckling behaviors of eccentrically stiffened functionally graded material (ES-FGM) plates on elastic foundations subjected to in-plane compressive loads or thermal loads are investigated by an analytical solution. The novelty of this work is that FGM plates are reinforced by FGM stiffeners and the temperature, stiffener, foundation are considered. The first-order shear deformation plate theory is used. The thermal elements of plate and stiffeners in fundamental equations are introduced. Theoretical formulations based on the smeared stiffeners technique and the first-order shear deformation plate theory, are derived. The analytical expressions to determine the static critical buckling load and post-buckling load-deflection curves are obtained.
\end{abstract}

Keywords: Stiffened plates, nonlinear analysis, functionally graded material (FGM), thermal environment, elastic foundation.

\section{INTRODUCTION}

Stiffened plates are structural components consisting of plates reinforced by a system of stiffeners to enhance their load carrying capacities. Stiffened plates nowadays are widely used in modern industry, such as ships, bridges, tank roofs, vehicles, etc. Many researches have been published regarding stiffened plates. Analysis of linear buckling of stiffened plates by the orthotropic plate method may be found in Timoshenko and Gere [1]. The elastic buckling and post-buckling behavior of eccentrically stiffened plate are evaluated analytically by Steen [2] using the simplified direct energy approach together with Marguerre's plate theory. Influence of stiffener location on the stability of stiffened plates under compression and in-plane bending is studied by Bedair [3].

For un-stiffened plates without foundation, many studies have been focused on the buckling behavior analysis under mechanical and thermal loading. Reddy [4] presented

(C) 2015 Vietnam Academy of Science and Technology 
the finite element formulation for linear and nonlinear thermo-mechanical bending response of plates made up of functionally graded material (FGM) using higher order shear deformation theory (HSDT). Lanhe [5] presented an analytical solution for the thermal buckling of FGM rectangular simply supported plate subjected to uniform temperature rise and gradient through the thickness of plate, using FSDT. The results on the nonlinear analysis of stability for functionally graded un-stiffened plates under mechanical and thermal loads using the classical plate theory (CPT) have been considered by Tung and Duc [6]. Achchhe Lal et al. [7] examined the second order statistics of post-buckling response of functionally graded materials plate subjected to mechanical and thermal loading with nonuniform temperature changes subjected to temperature independent and dependent material properties using HSDT with von-Karman nonlinear kinematic.

For plates resting on elastic foundations, many significant results on the buckling and post-buckling are obtained. Duc and Tung [8] presents an analytical investigation on the buckling and post-buckling behaviors of thick FGM un-stiffened plates resting on elastic foundations under in-plane compressive, thermal and thermomechanical loads based on HSDT taking into account Von Karman nonlinearity. Kiani and Eslami [9] studied analytically the buckling of heated functionally graded material annular plates on Pasternak-type elastic foundation based on the CPT. Naderi et al. [10] presented an exact analytical solution for buckling analysis of moderately thick functionally graded sector plates resting on Winkler elastic foundation according to FSDT.

As can be seen that the above introduced works only relate to un-stiffened FGM structures. Recently, Najafizadeh et al. [11] with the stability equation given in terms of displacement investigated the mechanical buckling behavior of FGM stiffened cylindrical shells reinforced by rings and stringers based on the classical shell theory. The stiffeners and skin are assumed to be made of FGM and its properties vary continuously through the thickness. Bich et al. $[12,13]$ investigated the nonlinear static buckling behavior of eccentrically stiffened imperfect FGM plates and shallow shells and the nonlinear dynamic response of eccentrically stiffened FGM imperfect panels and doubly curved thin shallow shells on the basis of the classical plate and shell theory. Stiffeners in these researches are assumed to be homogenous. The results on the static nonlinear buckling and post-buckling analysis of eccentrically stiffened FGM circular cylindrical shells under external pressure or torsional load are obtained Dung and Hoa [14,15], whereas the material properties of shell and stiffeners are assumed to be continuously graded in the thickness direction. Dung and Nam [16] presented nonlinear dynamic analysis of eccentrically stiffened functionally graded circular cylindrical thin shells surrounded by an elastic medium. Dung et al. [17] investigated the stability of functionally graded truncated conical shells surrounded by an elastic medium. Shells are reinforced by stringers and rings in which material properties of shell and stiffeners are graded in the thickness direction according to a volume fraction power-law distribution.

Recently, Duc et. al. $[18,19]$ investigated the nonlinear post-buckling of imperfect eccentrically stiffened thin FGM plates and FGM double curved thin shallow shells on elastic foundation in thermal environments with temperature-dependent material properties based on the classical plate theory and the classical shell theory, respectively. Dung and Hoa [20] presented the nonlinear buckling and post-buckling of functionally graded 
stiffened thin circular cylindrical shells under torsional load surrounded by elastic foundations in thermal environments based on the classical shell theory. The elastic medium in these articles is assumed as two-parameter elastic foundation model proposed by Pasternak.

In this paper, the buckling and post-buckling behaviors of eccentrically stiffened functionally graded material (ES-FGM) plates on elastic foundations subjected to inplane compressive loads or thermal loads are investigated by an analytical. We focus on three highlights as follows:

- FGM plates are reinforced by FGM stiffeners.

- The thermal element of stiffeners in $N_{i j}, M_{i j}$ is taken into account.

- The unknown functions $\phi_{x}$ and $\phi_{y}$ are chosen in the form of two terms, so we only need to apply Galerkin method one time for one resulting equation.

Three kinds of loads, namely, in-plane compressive, thermal and thermomechanical are considered. Theoretical formulations based on the smeared stiffeners technique and the first-order shear deformation plate theory, are derived. The analytical expressions to determine the static critical buckling load and analyze the post-buckling loaddeflection curves are obtained. The effects of thermal element, stiffeners, foundation, geometrical and material parameters are shown.

\section{THEORETICAL DERIVATIONS}

\subsection{Functionally graded materials}

Consider an eccentrically stiffened functionally graded (ES-FGM) rectangular plate of length $a$, width $b$, and uniform thickness $h$ resting on elastic foundations shown in Fig. 1. The plate is referred to Descartes coordinate system $(x, y, z)$ in which the plane Oxy coincides with the un-deformed middle surface of the plate and the axis $0 z$ is in the thickness direction $(-h / 2 \leq z \leq h / 2)$. The functionally graded material of plates is assumed to be varied continuously in the thickness direction and made from a mixture of ceramic and metal with the volume-fractions given by a power-law distribution as

$$
V_{m}+V_{c}=1, \quad V_{c}=V_{c}(z)=\left(\frac{z}{h}+\frac{1}{2}\right)^{k},
$$

where $k \geq 0$ is the volume fraction exponent, and the subscripts $m$ and $c$ refer to the metal and ceramic constituents, respectively.

Effective properties $\operatorname{Pr}_{\text {eff }}$ of FGM plate are determined by linear rule of mixtures as

$$
\operatorname{Pr}_{e f f}=\operatorname{Pr}_{m} V_{m}(z)+\operatorname{Pr}_{c} V_{c}(z),
$$

where $\operatorname{Pr}_{m}, \operatorname{Pr}_{c}$ are temperature-independent material properties of metal and ceramic constituent, respectively.

According to the mentioned law, Young's modulus, thermal expansion coefficient and thermal conductivity coefficient of FGM plate are of the form 


$$
\begin{aligned}
& E(z)=E_{m} V_{m}+E_{c} V_{c}=E_{m}+\left(E_{c}-E_{m}\right)\left(\frac{z}{h}+\frac{1}{2}\right)^{k}, \\
& \alpha(z)=\alpha_{m} V_{m}+\alpha_{c} V_{c}=\alpha_{m}+\left(\alpha_{c}-\alpha_{m}\right)\left(\frac{z}{h}+\frac{1}{2}\right)^{k}, \\
& K(z)=K_{m} V_{m}+K_{c} V_{c}=K_{m}+\left(K_{c}-K_{m}\right)\left(\frac{z}{h}+\frac{1}{2}\right)^{k},
\end{aligned}
$$

and Young's moduli, thermal expansion coefficients of FGM stiffeners are given by

$$
\begin{aligned}
& E_{s x}(z)=E_{m}+\left(E_{c}-E_{m}\right)\left(-\frac{2 z+h}{2 h_{1}}\right)^{k_{2}},-\frac{h}{2}-h_{1} \leq z \leq-\frac{h}{2} \\
& E_{s y}(z)=E_{m}+\left(E_{c}-E_{m}\right)\left(-\frac{2 z+h}{2 h_{2}}\right)^{k_{3}},-\frac{h}{2}-h_{2} \leq z \leq-\frac{h}{2} \\
& \alpha_{1}(z)=\alpha_{m}+\left(\alpha_{c}-\alpha_{m}\right)\left(-\frac{2 z+h}{2 h_{1}}\right)^{k_{2}},-\frac{h}{2}-h_{1} \leq z \leq-\frac{h}{2} \\
& \alpha_{2}(z)=\alpha_{m}+\left(\alpha_{c}-\alpha_{m}\right)\left(-\frac{2 z+h}{2 h_{2}}\right)^{k_{3}},-\frac{h}{2}-h_{2} \leq z \leq-\frac{h}{2}
\end{aligned}
$$

where $k_{2} \geq 0, k_{3} \geq 0$ are the volume fraction exponents; $E_{s x}(z), E_{s y}(z)$ and $\alpha_{1}(z), \alpha_{2}(z)$ are Young's moduli, and thermal expansion coefficients of $x$-direction and $y$-direction stiffeners, respectively.

The Poisson's ratio, in this work, is assumed to be a constant.

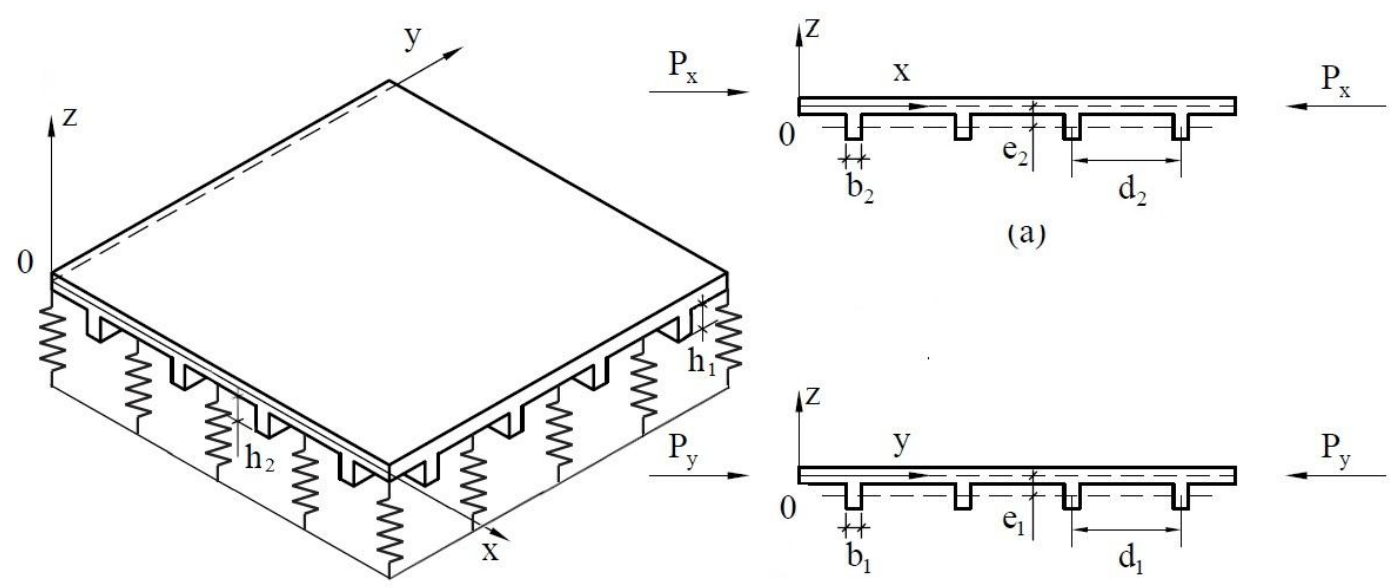

(b)

Fig. 1. Configuration of an eccentrically stiffened plate on elastic foundations 


\subsection{Basic relations and governing equations}

Denote $u, v, w$ being the displacement components of the mid-plane of the plate in $x, y, z$ directions respectively, and $\phi_{x}, \phi_{y}$ are the rotations of a transverse normal about the $y$ - and $x$-axes, respectively.

According to the first order shear deformation plate theory and geometrical nonlinearity in von Karman sense, the strain components across the plate thickness at a distance $z$ from the mid-plane are [5]

$$
\begin{aligned}
& {\left[\begin{array}{c}
\varepsilon_{x} \\
\varepsilon_{y} \\
\gamma_{x y}
\end{array}\right]=\left[\begin{array}{c}
\varepsilon_{x}^{0} \\
\varepsilon_{y}^{0} \\
\gamma_{x y}^{0}
\end{array}\right]+z\left[\begin{array}{c}
\kappa_{x} \\
\kappa_{y} \\
\kappa_{x y}
\end{array}\right],} \\
& {\left[\begin{array}{c}
\gamma_{x z} \\
\gamma_{y z}
\end{array}\right]=\left[\begin{array}{c}
\gamma_{x z}^{0} \\
\gamma_{y z}^{0}
\end{array}\right]=\left[\begin{array}{c}
w_{, x}+\phi_{x} \\
w_{, y}+\phi_{y}
\end{array}\right],}
\end{aligned}
$$

where

$$
\left[\begin{array}{c}
\varepsilon_{x}^{0} \\
\varepsilon_{y}^{0} \\
\gamma_{x y}^{0}
\end{array}\right]=\left[\begin{array}{c}
u_{, x}+\frac{1}{2} w_{, x}^{2} \\
v_{, y}+\frac{1}{2} w_{, y}^{2} \\
u_{, y}+v_{, x}+w_{, x} w_{, y}
\end{array}\right], \quad\left[\begin{array}{c}
\kappa_{x} \\
\kappa_{y} \\
\kappa_{x y}
\end{array}\right]=\left[\begin{array}{c}
\phi_{x, x} \\
\phi_{y, y} \\
\phi_{x, y}+\phi_{y, x}
\end{array}\right],
$$

in which $\varepsilon_{x}, \varepsilon_{y}$ are normal strains, $\gamma_{x y}$ is the in-plane shear strain and $\gamma_{x z}, \gamma_{y z}$ are the transverse shear deformations.

Using the relations (7), the geometric compatibility equation of plate is represented in the form

$$
\varepsilon_{x, y y}^{0}+\varepsilon_{y, x x}^{0}-\gamma_{x y, x y}^{0}=w_{, x y}^{2}-w_{, x x} w_{, y y} .
$$

The stress-strain relations taking into account the temperature for plate, are defined by Hooke's law as

$$
\begin{aligned}
& \left(\sigma_{x}^{p}, \sigma_{y}^{p}\right)=\frac{E(z)}{1-v^{2}}\left[\left(\varepsilon_{x}, \varepsilon_{y}\right)+v\left(\varepsilon_{y}, \varepsilon_{x}\right)\right]-\frac{E(z)}{1-v} \alpha(z) \Delta T(1,1), \quad \Delta T=T-T_{0}, \\
& \left(\sigma_{x y}^{p}, \sigma_{x z}^{p}, \sigma_{y z}^{p}\right)=\frac{E(z)}{2(1+v)}\left(\gamma_{x y}, \gamma_{x z}, \gamma_{y z}\right),
\end{aligned}
$$

and for stiffeners taking into account the temperature [21]

$$
\begin{aligned}
& \sigma_{x}^{s}=E_{s x}(z) \varepsilon_{x}-E_{s x}(z) \alpha_{1}(z) \Delta T, \quad \sigma_{y}^{s}=E_{s y}(z) \varepsilon_{y}-E_{s y}(z) \alpha_{2}(z) \Delta T, \\
& \sigma_{x z}^{s}=G_{s x}(z) \gamma_{x z}, \quad \sigma_{y z}^{s}=G_{s y}(z) \gamma_{y z},
\end{aligned}
$$

where the superscripts " $p$ " and "s" denote plate and stiffener respectively; $G_{s x}, G_{s y}$ are shear moduli of $x$-direction and $y$-direction stiffeners, respectively.

Taking into account the contribution of stiffeners by the smeared stiffeners technique and omitting the twist of stiffeners and integrating the above stress-strain equations and their moments through the thickness of the plate, we obtain the expressions for force, moment resultants and transverse shear force resultants of ES-FGM plate in 
thermal environment, as

$$
\begin{aligned}
& N_{x}=\left(A_{11}+\frac{E_{1 s} b_{1}}{d_{1}}\right) \varepsilon_{x}^{0}+A_{12} \varepsilon_{y}^{0}+\left(B_{11}+C_{1}\right) \phi_{x, x}+B_{12} \phi_{y, y}-\phi_{m}-\phi_{m x}, \\
& N_{y}=A_{12} \varepsilon_{x}^{0}+\left(A_{22}+\frac{E_{1 r} b_{2}}{d_{2}}\right) \varepsilon_{y}^{0}+B_{12} \phi_{x, x}+\left(B_{22}+C_{2}\right) \phi_{y, y}-\phi_{m}-\phi_{m y}, \\
& N_{x y}=A_{66} \gamma_{x y}^{0}+B_{66}\left(\phi_{x, y}+\phi_{y, x}\right) \\
& M_{x}=\left(B_{11}+C_{1}\right) \varepsilon_{x}^{0}+B_{12} \varepsilon_{y}^{0}+\left(D_{11}+\frac{E_{3 s} b_{1}}{d_{1}}\right) \phi_{x, x}+D_{12} \phi_{y, y}-\phi_{b}-\phi_{b x}, \\
& M_{y}=B_{12} \varepsilon_{x}^{0}+\left(B_{22}+C_{2}\right) \varepsilon_{y}^{0}+\left(D_{22}+\frac{E_{3 r} b_{2}}{d_{2}}\right) \phi_{y, y}+D_{12} \phi_{x, x}-\phi_{b}-\phi_{b y} \\
& M_{x y}=B_{66} \gamma_{x y}^{0}+D_{66}\left(\phi_{x, y}+\phi_{y, x}\right) \\
& \quad Q_{x}=A_{44} w_{, x}+A_{44} \phi_{x} \\
& \quad Q_{y}=A_{55} w_{, y}+A_{55} \phi_{y}
\end{aligned}
$$

where the coefficients $A_{i j}, B_{i j}, D_{i j}$ and the expressions $\phi_{m}, \phi_{m x}, \phi_{m y}, \phi_{b}, \phi_{b x}, \phi_{b y}$ are defined in Appendix I.

The relations (11) and (12) are the most significant contribution found in this work in which the thermal elements in plate and stiffeners in equations of $N_{i j}$ and $M_{i j}$ are considered.

The strain-force resultant relations are obtained reversely from Eq. (11)

$$
\begin{aligned}
& \varepsilon_{x}^{0}=A_{22}^{*} N_{x}-A_{12}^{*} N_{y}-B_{11}^{*} \phi_{x, x}-B_{12}^{*} \phi_{y, y}+\left(A_{22}^{*}-A_{12}^{*}\right) \phi_{m}+A_{22}^{*} \phi_{m x}-A_{12}^{*} \phi_{m y}, \\
& \varepsilon_{y}^{0}=A_{11}^{*} N_{y}-A_{12}^{*} N_{x}-B_{21}^{*} \phi_{x, x}-B_{22}^{*} \phi_{y, y}+\left(A_{11}^{*}-A_{12}^{*}\right) \phi_{m}+A_{11}^{*} \phi_{m y}-A_{12}^{*} \phi_{m x}, \\
& \gamma_{x y}^{0}=A_{66}^{*} N_{x y}-B_{66}^{*}\left(\phi_{x, y}+\phi_{y, x}\right) .
\end{aligned}
$$

Substituting Eq. (14) into Eq. (12) yields

$$
\begin{aligned}
& M_{x}=B_{11}^{*} N_{x}+B_{21}^{*} N_{y}+D_{11}^{*} \phi_{x, x}+D_{12}^{*} \phi_{y, y}+\left(B_{11}^{*}+B_{21}^{*}\right) \phi_{m}+B_{11}^{*} \phi_{m x}+B_{21}^{*} \phi_{m y}-\phi_{b}-\phi_{b x}, \\
& M_{y}=B_{12}^{*} N_{x}+B_{22}^{*} N_{y}+D_{21}^{*} \phi_{x, x}+D_{22}^{*} \phi_{y, y}+\left(B_{12}^{*}+B_{22}^{*}\right) \phi_{m}+B_{12}^{*} \phi_{m x}+B_{22}^{*} \phi_{m y}-\phi_{b}-\phi_{b y}, \\
& M_{x y}=B_{66}^{*} N_{x y}+D_{66}^{*}\left(\phi_{x, y}+\phi_{y, x}\right),
\end{aligned}
$$

where the coefficients $A_{i j}^{*}, B_{i j}^{*}$ and $D_{i j}^{*}$ are given in Appendix II.

The nonlinear equilibrium equations of a perfect FGM plate on elastic foundations based on the first order shear deformation theory are $[5,22]$

$$
\begin{gathered}
N_{x, x}+N_{x y, y}=0, \\
N_{x y, x}+N_{y, y}=0, \\
Q_{x, x}+Q_{y, y}+N_{x} w_{, x x}+2 N_{x y} w_{, x y}+N_{y} w_{, y y}+q-K_{1} w+K_{2}\left(w_{, x x}+w_{, y y}\right)=0, \\
M_{x, x}+M_{x y, y}-Q_{x}=0, \\
M_{x y, x}+M_{y, y}-Q_{y}=0,
\end{gathered}
$$

where $q$ is an uniform transversal force, $K_{1}\left(\mathrm{~N} / \mathrm{m}^{3}\right)$ is Winkler foundation modulus and $K_{2}(\mathrm{~N} / \mathrm{m})$ is the shear layer foundation stiffness of Pasternak model. 
By putting

$$
N_{x}=f_{, y y}, \quad N_{y}=f_{, x x}, \quad N_{x y}=-f_{, x y}
$$

it is easy to see that the first two equations (16) and (17) are automatically satisfied, and three resting equations become

$$
\begin{aligned}
& M_{x, x x}+2 M_{x y, x y}+M_{y, y y}+f_{, y y} w_{, x x}-2 f_{, x y} w_{, x y}+f_{, x x} w_{, y y}+q-K_{1} w+K_{2}\left(w_{, x x}+w_{, y y}\right)=0, \\
& M_{x, x}+M_{x y, y}-Q_{x}=0 \\
& M_{x y, x}+M_{y, y}-Q_{y}=0 .
\end{aligned}
$$

Substituting the expressions of $M_{i j}$ from Eq. (15) and $Q_{x}, Q_{y}$ from (13) into Eq. (22), we obtain

$$
\begin{gathered}
B_{21}^{*} \frac{\partial^{4} f}{\partial x^{4}}+\left(B_{11}^{*}+B_{22}^{*}-2 B_{66}^{*}\right) \frac{\partial^{4} f}{\partial x^{2} \partial y^{2}}+B_{12}^{*} \frac{\partial^{4} f}{\partial y^{4}}+D_{11}^{*} \frac{\partial^{3} \phi_{x}}{\partial x^{3}}+ \\
+\left(D_{12}^{*}+2 D_{66}^{*}\right) \frac{\partial^{3} \phi_{y}}{\partial x^{2} \partial y}+\left(D_{21}^{*}+2 D_{66}^{*}\right) \frac{\partial^{3} \phi_{x}}{\partial x \partial y^{2}}+D_{22}^{*} \frac{\partial^{3} \phi_{y}}{\partial y^{3}}+\frac{\partial^{2} f}{\partial y^{2}} \frac{\partial^{2} w}{\partial x^{2}}+\quad(23) \\
-2 \frac{\partial^{2} f}{\partial x \partial y} \frac{\partial^{2} w}{\partial x \partial y}+\frac{\partial^{2} f}{\partial x^{2}} \frac{\partial^{2} w}{\partial y^{2}}+q-K_{1} w+K_{2}\left(\frac{\partial^{2} w}{\partial x^{2}}+\frac{\partial^{2} w}{\partial y^{2}}\right)=0, \\
B_{21}^{*} \frac{\partial^{3} f}{\partial x^{3}}+\left(B_{11}^{*}-B_{66}^{*}\right) \frac{\partial^{3} f}{\partial x \partial y^{2}}+D_{11}^{*} \frac{\partial^{2} \phi_{x}}{\partial x^{2}}+\left(D_{12}^{*}+D_{66}^{*}\right) \frac{\partial^{2} \phi_{y}}{\partial x \partial y}+D_{66}^{*} \frac{\partial^{2} \phi_{x}}{\partial y^{2}}-A_{44} \frac{\partial w}{\partial x}-A_{44} \phi_{x}=0, \\
B_{12}^{*} \frac{\partial^{3} f}{\partial y^{3}}+\left(B_{22}^{*}-B_{66}^{*}\right) \frac{\partial^{3} f}{\partial y \partial x^{2}}+D_{22}^{*} \frac{\partial^{2} \phi_{y}}{\partial y^{2}}+\left(D_{21}^{*}+D_{66}^{*}\right) \frac{\partial^{2} \phi_{x}}{\partial x \partial y}+D_{66}^{*} \frac{\partial^{2} \phi_{y}}{\partial x^{2}}-A_{55} \frac{\partial w}{\partial y}-A_{55} \phi_{y}=0 .
\end{gathered}
$$

The three coupled equations (23), (24) and (25) include four unknown functions $w, \phi_{x}, \phi_{y}$ and $f$ so it is necessary to find a fourth equation relating to these functions by using the compatibility equation (8). For this aim, substituting the expressions of Eq. (14) into Eq. (8), one can write as

$$
\begin{gathered}
A_{11}^{*} \frac{\partial^{4} f}{\partial x^{4}}+\left(A_{66}^{*}-2 A_{12}^{*}\right) \frac{\partial^{4} f}{\partial x^{2} \partial y^{2}}+A_{22}^{*} \frac{\partial^{4} f}{\partial y^{4}}-B_{21}^{*} \frac{\partial^{3} \phi_{x}}{\partial x^{3}}-\left(B_{11}^{*}-B_{66}^{*}\right) \frac{\partial^{3} \phi_{x}}{\partial x \partial y^{2}}+ \\
-\left(B_{22}^{*}-B_{66}^{*}\right) \frac{\partial^{3} \phi_{y}}{\partial y \partial x^{2}}-B_{12}^{*} \frac{\partial^{3} \phi_{y}}{\partial y^{3}}-\left(\frac{\partial^{2} w}{\partial x \partial y}\right)^{2}+\frac{\partial^{2} w}{\partial x^{2}} \frac{\partial^{2} w}{\partial y^{2}}=0 .
\end{gathered}
$$

For initial imperfect ES-FGM plates: The initial imperfection of the plate considered here can be seen as a small deviation of the plate middle surface from the perfect shape and assume that it is very small compared with the thickness of the plate. Let $w^{*}=$ $w^{*}(x, y)$ is a known function representing initial imperfection of the plate. Two equations 
(23) and (26) are modified into form as

$$
\begin{aligned}
& B_{21}^{*} \frac{\partial^{4} f}{\partial x^{4}}+\left(B_{11}^{*}+B_{22}^{*}-2 B_{66}^{*}\right) \frac{\partial^{4} f}{\partial x^{2} \partial y^{2}}+B_{12}^{*} \frac{\partial^{4} f}{\partial y^{4}}+D_{11}^{*} \frac{\partial^{3} \phi_{x}}{\partial x^{3}}+\left(D_{12}^{*}+2 D_{66}^{*}\right) \frac{\partial^{3} \phi_{y}}{\partial x^{2} \partial y} \\
& +\left(D_{21}^{*}+2 D_{66}^{*}\right) \frac{\partial^{3} \phi_{x}}{\partial x \partial y^{2}}+D_{22}^{*} \frac{\partial^{3} \phi_{y}}{\partial y^{3}}+\frac{\partial^{2} f}{\partial y^{2}}\left(\frac{\partial^{2} w}{\partial x^{2}}+\frac{\partial^{2} w^{*}}{\partial x^{2}}\right)-2 \frac{\partial^{2} f}{\partial x \partial y}\left(\frac{\partial^{2} w}{\partial x \partial y}+\frac{\partial^{2} w^{*}}{\partial x \partial y}\right) \\
& +\frac{\partial^{2} f}{\partial x^{2}}\left(\frac{\partial^{2} w}{\partial y^{2}}+\frac{\partial^{2} w^{*}}{\partial y^{2}}\right)+q-K_{1} w+K_{2}\left(\frac{\partial^{2} w}{\partial x^{2}}+\frac{\partial^{2} w}{\partial y^{2}}\right)=0 \\
& A_{11}^{*} \frac{\partial^{4} f}{\partial x^{4}}+\left(A_{66}^{*}-2 A_{12}^{*}\right) \frac{\partial^{4} f}{\partial x^{2} \partial y^{2}}+A_{22}^{*} \frac{\partial^{4} f}{\partial y^{4}}-B_{21}^{*} \frac{\partial^{3} \phi_{x}}{\partial x^{3}}-\left(B_{11}^{*}-B_{66}^{*}\right) \frac{\partial^{3} \phi_{x}}{\partial x \partial y^{2}}-\left(B_{22}^{*}-B_{66}^{*}\right) \frac{\partial^{3} \phi_{y}}{\partial y \partial x^{2}} \\
& -B_{12}^{*} \frac{\partial^{3} \phi_{y}}{\partial y^{3}}-\left(\frac{\partial^{2} w}{\partial x \partial y}\right)^{2}+\frac{\partial^{2} w}{\partial x^{2}} \frac{\partial^{2} w}{\partial y^{2}}-2 \frac{\partial^{2} w}{\partial x \partial y} \frac{\partial^{2} w^{*}}{\partial x \partial y}+\frac{\partial^{2} w}{\partial x^{2}} \frac{\partial^{2} w^{*}}{\partial y^{2}}+\frac{\partial^{2} w}{\partial y^{2}} \frac{\partial^{2} w^{*}}{\partial x^{2}}=0,
\end{aligned}
$$

and two Eqs. (24) and (25) are unchanged as

$$
\begin{aligned}
& B_{21}^{*} \frac{\partial^{3} f}{\partial x^{3}}+\left(B_{11}^{*}-B_{66}^{*}\right) \frac{\partial^{3} f}{\partial x \partial y^{2}}+D_{11}^{*} \frac{\partial^{2} \phi_{x}}{\partial x^{2}}+\left(D_{12}^{*}+D_{66}^{*}\right) \frac{\partial^{2} \phi_{y}}{\partial x \partial y}+D_{66}^{*} \frac{\partial^{2} \phi_{x}}{\partial y^{2}}-A_{44} \frac{\partial w}{\partial x}-A_{44} \phi_{x}=0 \\
& B_{12}^{*} \frac{\partial^{3} f}{\partial y^{3}}+\left(B_{22}^{*}-B_{66}^{*}\right) \frac{\partial^{3} f}{\partial y \partial x^{2}}+D_{22}^{*} \frac{\partial^{2} \phi_{y}}{\partial y^{2}}+\left(D_{21}^{*}+D_{66}^{*}\right) \frac{\partial^{2} \phi_{x}}{\partial x \partial y}+D_{66}^{*} \frac{\partial^{2} \phi_{y}}{\partial x^{2}}-A_{55} \frac{\partial w}{\partial y}-A_{55} \phi_{y}=0 .
\end{aligned}
$$

Eqs. $(23) \div(26)$ or $(27) \div(30)$ are nonlinear equations in terms of four unknown functions $w, \phi_{x}, \phi_{y}$ and $f$ and are used to investigate the buckling and post-buckling of perfect or imperfect eccentrically stiffened functionally graded (ES-FGM) plates subjected to mechanical load, thermal load or combined thermo-mechanical loads. It is obvious that this system of equations is more complicated than the one established by using the classical plate theory. This is also the main reason why the nonlinear stability analysis of stiffened FGM plate based on FSDT is much more complicated than nonlinear stability analysis of stiffened FGM plate based on CPT (there are only two nonlinear equations).

\section{BOUNDARY CONDITIONS AND PROCEDURE OF THE SOLUTION}

Suppose that three cases of boundary conditions for an imperfect ES-FGM plate will be considered [8]

Case (1). Four edges of plate are simply supported and freely movable (FM) i.e.

$$
\begin{array}{llll}
w=\phi_{y}=N_{x y}=M_{x}=0, & N_{x}=N_{x 0} & \text { at } & x=0, a, \\
w=\phi_{x}=N_{x y}=M_{y}=0, & N_{y}=N_{y 0} & \text { at } & y=0, b .
\end{array}
$$

Case (2). Four edges of plate are simply supported and immovable (IM) i.e.

$$
\begin{aligned}
& w=u=\phi_{y}=M_{x}=0, \quad N_{x}=N_{x 0} \quad \text { at } \quad x=0, a, \\
& w=v=\phi_{x}=M_{y}=0, \quad N_{y}=N_{y 0} \quad \text { at } \quad y=0, b \text {. }
\end{aligned}
$$


Case (3). The edges of plate are simply supported. Uniaxial edge loads are applied in the direction of the $x$-coordinate. The edges $x=0, x=a$ are considered freely movable, the remaining two edges being unloaded and immovable. The boundary conditions, for this case, are

$$
\begin{aligned}
& w=\phi_{y}=N_{x y}=M_{x}=0, \quad N_{x}=N_{x 0} \quad \text { at } \quad x=0, a, \\
& w=v=\phi_{x}=M_{y}=0, \quad N_{y}=N_{y 0} \quad \text { at } \quad y=0, b .
\end{aligned}
$$

where $N_{x 0}, N_{y 0}$ are pre-buckling force resultants in the direction $x$ and $y$ respectively, for case (1) and the first of case (3), and they are fictitious compressive edge loads rendering the edges immovable for case (2) and the second of case (3).

The analytical solution of the system of Eqs. (27) $\div(30)$ satisfying the boundary conditions exactly for $w$ and on average sense for $\phi_{x}, \phi_{y}$ can be found in the form [23]

$$
\begin{gathered}
w=W \sin \alpha x \sin \beta y, w^{*}=\xi h \sin \alpha x \sin \beta y, \\
\phi_{x}=\phi_{10} \cos \alpha x \sin \beta y+\phi_{11} \sin 2 \alpha x, \phi_{y}=\phi_{20} \sin \alpha x \cos \beta y+\phi_{21} \sin 2 \beta y, \\
f=f_{1} \cos 2 \alpha x+f_{2} \cos 2 \beta y+f_{3} \sin \alpha x \sin \beta y+\frac{1}{2} N_{x 0} y^{2}+\frac{1}{2} N_{y 0} x^{2},
\end{gathered}
$$

where $\alpha=\frac{m \pi}{a}, \beta=\frac{n \pi}{b}$ and $m, n$ are numbers of half waves in $x$ and $y$ directions, respectively, and the coefficient $\xi \in[0,1]$ is an imperfection size of plate.

Substituting Eq. (34) into Eqs. (28), (29) and (30) and carrying out some calculations, yield

$$
f_{1}=L_{1} \cdot W \cdot(W+2 \xi h), \quad f_{2}=L_{2} \cdot W \cdot(W+2 \xi h), f_{3}=L_{3} \cdot W,
$$

and

$$
\phi_{10}=L_{4} \cdot W, \phi_{20}=L_{5} \cdot W, \phi_{11}=L_{6} \cdot W \cdot(W+2 \xi h), \phi_{21}=L_{7} \cdot W \cdot(W+2 \xi h),
$$

where the coefficients $L_{i}$ are defined in Appendix III.

Substituting the expression (34) into Eq. (27) and applying Galerkin's method for resulting equation, we obtain

$$
\begin{aligned}
& {\left[\left(16 \alpha^{4} B_{21}^{*} L_{1}+16 \beta^{4} B_{12}^{*} L_{2}-8 \alpha^{3} D_{11}^{*} L_{6}-8 \beta^{3} D_{22}^{*} L_{7}\right) \cdot W \cdot(W+2 \xi h)+\right.} \\
& \left.-2 \alpha^{2} \beta^{2} L_{3} \cdot W \cdot(W+\xi h)\right]\left(\frac{-16 \delta_{m} \delta_{n}}{3 \alpha \beta a b}\right)-2 \alpha^{2} \beta^{2}\left(L_{1}+L_{2}\right) \cdot W \cdot(W+\xi h) \cdot(W+2 \xi h)+ \\
& +\left\{\left[\alpha^{4} B_{21}^{*}+\alpha^{2} \beta^{2}\left(B_{11}^{*}+B_{22}^{*}-2 B_{66}^{*}\right)+\beta^{4} B_{12}^{*}\right] L_{3}+\left[\alpha^{3} D_{11}^{*}+\alpha \beta^{2}\left(D_{21}^{*}+2 D_{66}^{*}\right)\right] L_{4}+\right. \\
& \left.+\left[\beta^{3} D_{22}^{*}+\alpha^{2} \beta\left(D_{12}^{*}+2 D_{66}^{*}\right)\right] L_{5}-K_{1}-\left(\alpha^{2}+\beta^{2}\right) K_{2}\right\} \cdot W \\
& -\left(\alpha^{2} N_{x 0}+\beta^{2} N_{y 0}\right) \cdot(W+\xi h)+q \frac{16 \delta_{m} \delta_{n}}{\alpha \beta a b}=0,
\end{aligned}
$$

in which $\delta_{m}=\frac{1-(-1)^{m}}{2}, \delta_{n}=\frac{1-(-1)^{n}}{2}$.

Nonlinear Eq. (37) is used to determine buckling loads and load-deflection postbuckling curves of imperfect ES-FGM plates subjected to mechanical compressive loads, thermal and combined loads and on elastic foundations. 


\section{BUCKLING AND POST-BUCKLING ANALYSIS}

\subsection{Mechanical stability analysis}

Consider a rectangular imperfect ES-FGM plate being simply supported at its edges and freely movable (Case (1)) and subjected to the in-plane compressive loads $P_{x}$ and $P_{y}$ uniformly distributed along the edges $x=0, a$ and $y=0, b$ respectively.

If $q=0, N_{x 0}=-h P_{x}, N_{y 0}=-h P_{y}$ and putting $\lambda=\frac{P_{y}}{P_{x}}, \bar{W}=\frac{W}{h}$, Eq. (37) leads to the explicit relation

$$
\begin{aligned}
P_{x}= & \frac{-1}{h\left(\alpha^{2}+\lambda \beta^{2}\right)}\left\{\left[\left(16 \alpha^{4} B_{21}^{*} L_{1}+16 \beta^{4} B_{12}^{*} L_{2}-8 \alpha^{3} D_{11}^{*} L_{6}-8 \beta^{3} D_{22}^{*} L_{7}\right) \cdot \frac{\bar{W} \cdot(\bar{W}+2 \xi)}{\bar{W}+\xi}\right.\right. \\
& \left.-2 \alpha^{2} \beta^{2} L_{3} \cdot \bar{W}\right]\left(\frac{-16 h \delta_{m} \delta_{n}}{3 \alpha \beta a b}\right)-2 \alpha^{2} \beta^{2}\left(L_{1}+L_{2}\right) h^{2} \cdot \bar{W} \cdot(\bar{W}+2 \xi) \\
& +\left\{\left[\alpha^{4} B_{21}^{*}+\alpha^{2} \beta^{2}\left(B_{11}^{*}+B_{22}^{*}-2 B_{66}^{*}\right)+\beta^{4} B_{12}^{*}\right] L_{3}+\left[\alpha^{3} D_{11}^{*}+\alpha \beta^{2}\left(D_{21}^{*}+2 D_{66}^{*}\right)\right] L_{4}\right. \\
& \left.\left.+\left[\beta^{3} D_{22}^{*}+\alpha^{2} \beta\left(D_{12}^{*}+2 D_{66}^{*}\right)\right] L_{5}-K_{1}-\left(\alpha^{2}+\beta^{2}\right) K_{2}\right\} \frac{\bar{W}}{\bar{W}+\xi}\right\} .
\end{aligned}
$$

For a perfect ES-FGM plate, $\xi=0$, Eq. (38) becomes

$$
\begin{aligned}
P_{x}= & \frac{-1}{h\left(\alpha^{2}+\lambda \beta^{2}\right)}\left\{\left(16 \alpha^{4} B_{21}^{*} L_{1}+16 \beta^{4} B_{12}^{*} L_{2}-8 \alpha^{3} D_{11}^{*} L_{6}-8 \beta^{3} D_{22}^{*} L_{7}\right.\right. \\
& \left.-2 \alpha^{2} \beta^{2} L_{3}\right)\left(\frac{-16 h \delta_{m} \delta_{n}}{3 \alpha \beta a b}\right) \cdot \bar{W}-2 \alpha^{2} \beta^{2}\left(L_{1}+L_{2}\right) h^{2} \cdot \bar{W}^{2} \\
& +\left[\alpha^{4} B_{21}^{*}+\alpha^{2} \beta^{2}\left(B_{11}^{*}+B_{22}^{*}-2 B_{66}^{*}\right)+\beta^{4} B_{12}^{*}\right] L_{3}+\left[\alpha^{3} D_{11}^{*}\right. \\
& \left.\left.+\alpha \beta^{2}\left(D_{21}^{*}+2 D_{66}^{*}\right)\right] L_{4}+\left[\beta^{3} D_{22}^{*}+\alpha^{2} \beta\left(D_{12}^{*}+2 D_{66}^{*}\right)\right] L_{5}-K_{1}-\left(\alpha^{2}+\beta^{2}\right) K_{2}\right\} .
\end{aligned}
$$

Taking $\bar{W} \rightarrow 0$, Eq. (39) gives the upper buckling load for a perfect ES-FGM plate

$$
P_{x}=\frac{-1}{h\left(\alpha^{2}+\lambda \beta^{2}\right)}\left\{\begin{array}{c}
{\left[\alpha^{4} B_{21}^{*}+\alpha^{2} \beta^{2}\left(B_{11}^{*}+B_{22}^{*}-2 B_{66}^{*}\right)+\beta^{4} B_{12}^{*}\right] L_{3}} \\
+\left[\alpha^{3} D_{11}^{*}+\alpha \beta^{2}\left(D_{21}^{*}+2 D_{66}^{*}\right)\right] L_{4} \\
+\left[\beta^{3} D_{22}^{*}+\alpha^{2} \beta\left(D_{12}^{*}+2 D_{66}^{*}\right)\right] L_{5}-K_{1}-\left(\alpha^{2}+\beta^{2}\right) K_{2}
\end{array}\right\} .
$$

Similarly, the upper static buckling load for ES-FGM plate based on the CPT, without foundation and only subjected to mechanical loads, can be determined [12, Eq. 36]

$$
P_{x \text { upper }}=\frac{\pi^{2}}{m^{2} a^{2} h}\left[\begin{array}{l}
D_{11}^{*} m^{4}+D_{22}^{*} n^{4} \frac{a^{4}}{b^{4}}+\left(D_{12}^{*}+D_{21}^{*}+4 D_{66}^{*}\right) m^{2} n^{2} \frac{a^{2}}{b^{2}} \\
+\frac{\left(B_{21}^{*} m^{4}+\left(B_{11}^{*}+B_{22}^{*}-2 B_{66}^{*}\right) m^{2} n^{2} \frac{a^{2}}{b^{2}}+B_{12}^{*} n^{4} \frac{a^{4}}{b^{4}}\right)^{2}}{A_{11}^{*} m^{4}+\left(A_{66}^{*}-2 A_{12}^{*}\right) m^{2} n^{2} \frac{a^{2}}{b^{2}}+A_{22}^{*} n^{4} \frac{a^{4}}{b^{4}}}
\end{array}\right] .
$$

\subsection{Thermal stability analysis}

Suppose that an imperfect ES-FGM plate on elastic foundations is a simple supported with immovable edges (Case (2)). So the condition expressing the immovable on 
the edges $u=0$ at $x=0, x=a$ and $v=0$ at $y=0, y=b$ is fulfilled on the average sense as $[24,25]$

$$
\int_{0}^{b} \int_{0}^{a} \frac{\partial u}{\partial x} d x d y=0, \quad \int_{0}^{a} \int_{0}^{b} \frac{\partial v}{\partial y} d y d x=0 .
$$

From the relations (7) and (14) we obtain the following equations

$$
\begin{aligned}
& u_{, x}=A_{22}^{*} f_{, y y}-A_{12}^{*} f_{, x x}-B_{11}^{*} \phi_{x, x}-B_{12}^{*} \phi_{y, y}+\left(A_{22}^{*}-A_{12}^{*}\right) \phi_{m}+A_{22}^{*} \phi_{m x}-A_{12}^{*} \phi_{m y}-\frac{1}{2} w_{, x}^{2}-w_{, x} w_{, x}^{*}, \\
& v_{, y}=A_{11}^{*} f_{, x x}-A_{12}^{*} f_{, y y}-B_{21}^{*} \phi_{x, x}-B_{22}^{*} \phi_{y, y}+\left(A_{11}^{*}-A_{12}^{*}\right) \phi_{m}+A_{11}^{*} \phi_{m y}-A_{12}^{*} \phi_{m x}-\frac{1}{2} w_{, y}^{2}-w_{, y} w_{, y}^{*} .
\end{aligned}
$$

Substituting expressions of (34) into Eq. (43), and then into the conditions (42), gives us

$$
\begin{aligned}
N_{x 0}= & {\left[\beta^{2} L_{3}-\alpha \Delta\left(A_{11}^{*} B_{11}^{*}+A_{12}^{*} B_{21}^{*}\right) L_{4}-\beta \Delta\left(A_{11}^{*} B_{12}^{*}+A_{12}^{*} B_{22}^{*}\right) L_{5}\right] W\left(\frac{4 \delta_{m} \delta_{n}}{\alpha \beta a b}\right) } \\
& +\frac{\Delta}{8}\left(\alpha^{2} A_{11}^{*}+\beta^{2} A_{12}^{*}\right) W(W+2 \xi h)-\phi_{m}-\phi_{m x}, \\
N_{y 0}= & {\left[\alpha^{2} L_{3}-\alpha \Delta\left(A_{12}^{*} B_{11}^{*}+A_{22}^{*} B_{21}^{*}\right) L_{4}-\beta \Delta\left(A_{12}^{*} B_{12}^{*}+A_{22}^{*} B_{22}^{*}\right) L_{5}\right] W\left(\frac{4 \delta_{m} \delta_{n}}{\alpha \beta a b}\right) } \\
& +\frac{\Delta}{8}\left(\alpha^{2} A_{12}^{*}+\beta^{2} A_{22}^{*}\right) W(W+2 \xi h)-\phi_{m}-\phi_{m y} .
\end{aligned}
$$

Introducing the relation (44) into Eq. (37), and $q=0$, we get

$$
\begin{aligned}
& \left(\alpha^{2}+\beta^{2}\right) \phi_{m}+\alpha^{2} \phi_{m x}+\beta^{2} \phi_{m y}=\left(t_{1} \frac{W \cdot(W+2 \xi h)}{W+\xi h}-t_{2} W\right)\left(\frac{16 \delta_{m} \delta_{n}}{3 \alpha \beta a b}\right) \\
& +\left(t_{3}+t_{30}\right) \cdot W \cdot(W+2 \xi h)-t_{4} \frac{W}{W+\xi h}+\left(t_{2}-t_{20}-t_{21}\right) W\left(\frac{4 \delta_{m} \delta_{n}}{\alpha \beta a b}\right),
\end{aligned}
$$

where

$$
\begin{aligned}
t_{1}= & 16 \alpha^{4} B_{21}^{*} L_{1}+16 \beta^{4} B_{12}^{*} L_{2}-8 \alpha^{3} D_{11}^{*} L_{6}-8 \beta^{3} D_{22}^{*} L_{7}, \\
t_{2}= & 2 \alpha^{2} \beta^{2} L_{3}, t_{3}=2 \alpha^{2} \beta^{2}\left(L_{1}+L_{2}\right), \\
t_{4}= & {\left[\alpha^{4} B_{21}^{*}+\alpha^{2} \beta^{2}\left(B_{11}^{*}+B_{22}^{*}-2 B_{66}^{*}\right)+\beta^{4} B_{12}^{*}\right] L_{3}+\left[\alpha^{3} D_{11}^{*}+\alpha \beta^{2}\left(D_{21}^{*}+2 D_{66}^{*}\right)\right] L_{4} } \\
& +\left[\beta^{3} D_{22}^{*}+\alpha^{2} \beta\left(D_{12}^{*}+2 D_{66}^{*}\right)\right] L_{5}-K_{1}-\left(\alpha^{2}+\beta^{2}\right) K_{2}, \\
t_{30}= & \frac{\Delta}{8}\left(\alpha^{4} A_{11}^{*}+2 \alpha^{2} \beta^{2} A_{12}^{*}+\beta^{4} A_{22}^{*}\right), \\
t_{20}= & \alpha \Delta\left[\left(\alpha^{2} A_{11}^{*}+\beta^{2} A_{12}^{*}\right) B_{11}^{*}+\left(\alpha^{2} A_{12}^{*}+\beta^{2} A_{22}^{*}\right) B_{21}^{*}\right] L_{4}, \\
t_{21}= & \beta \Delta\left[\left(\alpha^{2} A_{11}^{*}+\beta^{2} A_{12}^{*}\right) B_{12}^{*}+\left(\alpha^{2} A_{12}^{*}+\beta^{2} A_{22}^{*}\right) B_{22}^{*}\right] L_{5} .
\end{aligned}
$$

The equation (45) shows the explicit relationship of temperature-deflection of an imperfect ES-FGM plate on elastic foundations under thermal load. 


\subsubsection{Uniform temperature rise}

In this case, the FGM plate is exposed to temperature environment uniformly raised from initial value $T_{i}$ to final one $T_{f}$ and $\Delta T=T_{f}-T_{i}$ is a constant. Then the thermal parameters $\phi_{m}, \phi_{m x}, \phi_{m y}$ are given as

$$
\begin{aligned}
& \phi_{m}=\frac{h \cdot \Delta T}{1-v} P, \\
& \phi_{m x}=\frac{b_{1} h_{1}}{d_{1}}\left[E_{m} \alpha_{m}+\frac{E_{m} \alpha_{c m}+E_{c m} \alpha_{m}}{k_{2}+1}+\frac{E_{c m} \alpha_{c m}}{2 k_{2}+1}\right] \Delta T=\phi_{m x}^{0} \Delta T, \\
& \phi_{m y}=\frac{b_{2} h_{2}}{d_{2}}\left[E_{m} \alpha_{m}+\frac{E_{m} \alpha_{c m}+E_{c m} \alpha_{m}}{k_{3}+1}+\frac{E_{c m} \alpha_{c m}}{2 k_{3}+1}\right] \Delta T=\phi_{m y}^{0} \Delta T,
\end{aligned}
$$

where

$$
\begin{aligned}
& P=E_{m} \alpha_{m}+\frac{E_{m} \alpha_{c m}+E_{c m} \alpha_{m}}{k+1}+\frac{E_{c m} \alpha_{c m}}{2 k+1}, \\
& \phi_{m x}^{0}=\frac{b_{1} h_{1}}{d_{1}}\left(E_{m} \alpha_{m}+\frac{E_{m} \alpha_{c m}+E_{c m} \alpha_{m}}{k_{2}+1}+\frac{E_{c m} \alpha_{c m}}{2 k_{2}+1}\right), \\
& \phi_{m y}^{0}=\frac{b_{2} h_{2}}{d_{2}}\left(E_{m} \alpha_{m}+\frac{E_{m} \alpha_{c m}+E_{c m} \alpha_{m}}{k_{3}+1}+\frac{E_{c m} \alpha_{c m}}{2 k_{3}+1}\right) .
\end{aligned}
$$

Combining Eq. (45) with Eq. (47) and putting $\bar{W}=\frac{W}{h}$ leads to

$$
\Delta T=\frac{1}{\frac{h\left(\alpha^{2}+\beta^{2}\right)}{1-v} P+\alpha^{2} \phi_{m x}^{0}+\beta^{2} \phi_{m y}^{0}}\left\{\begin{array}{c}
{\left[t_{1} \frac{\bar{W} \cdot(\bar{W}+2 \xi)}{\bar{W}+\xi}-t_{2} \cdot \bar{W}\right]\left(\frac{16 h \delta_{m} \delta_{n}}{3 \alpha \beta a b}\right)} \\
+\left(t_{3}+t_{30}\right) h^{2} \cdot \bar{W} \cdot(\bar{W}+2 \xi)-t_{4} \frac{\bar{W}}{\bar{W}+\xi} \\
+\left(t_{2}-t_{20}-t_{21}\right) \bar{W}\left(\frac{4 h \delta_{m} \delta_{n}}{\alpha \beta a b}\right)
\end{array}\right\} .
$$

If the imperfection $\xi=0$ and $\bar{W} \rightarrow 0$, Eq. (49) gives us

$$
\Delta T=\frac{-\left\{\begin{array}{c}
{\left[\alpha^{4} B_{21}^{*}+\alpha^{2} \beta^{2}\left(B_{11}^{*}+B_{22}^{*}-2 B_{66}^{*}\right)+\beta^{4} B_{12}^{*}\right] L_{3}} \\
+\left[\alpha^{3} D_{11}^{*}++\alpha \beta^{2}\left(D_{21}^{*}+2 D_{66}^{*}\right)\right] L_{4} \\
+\left[\beta^{3} D_{22}^{*}+\alpha^{2} \beta\left(D_{12}^{*}+2 D_{66}^{*}\right)\right] L_{5}-K_{1}-\left(\alpha^{2}+\beta^{2}\right) K_{2}
\end{array}\right\}}{\frac{h\left(\alpha^{2}+\beta^{2}\right)}{1-v} P+\alpha^{2} \phi_{m x}^{0}+\beta^{2} \phi_{m y}^{0}} .
$$

4.2.2. Nonlinear temperature change across the thickness

Assume that the temperature through thickness is governed by the one-dimensional Fourier equation of steady-state heat conduction

$$
\frac{d}{d z}\left[K(z) \frac{d T}{d z}\right]=0,\left.\quad T\right|_{z=h / 2}=T_{c},\left.\quad T\right|_{z=-h / 2}=T_{m},
$$

where $T_{m}$ and $T_{c}$ are temperatures at metal-rich and ceramic-rich surfaces, respectively. By solving Eq. (51) with mentioned boundary conditions, the solution for temperature 
distribution across the plate thickness is obtained

$$
T(z)=T_{m}+\Delta T \frac{\sum_{n=0}^{\infty} \frac{1}{k n+1}\left(\frac{-K_{c m}}{K_{m}}\right)^{n}\left(\frac{z}{h}+\frac{1}{2}\right)^{k n+1}}{\sum_{n=0}^{\infty} \frac{1}{k n+1}\left(\frac{-K_{c m}}{K_{m}}\right)^{n}}
$$

in which $\Delta T=T_{c}-T_{m}$.

In only this subsection, we consider homogenous stiffeners, by following the same procedure as in the subsection 4.2.1, the expressions of the thermal parameter, in this case, are

$$
\phi_{m}=\frac{\Delta T}{1-v} . H, \quad \phi_{m x}=\frac{b_{1}}{d_{1}} E_{s x} \alpha_{1} \Delta T . H_{x}, \quad \phi_{m y}=\frac{b_{2}}{d_{2}} E_{s y} \alpha_{2} \Delta T . H_{y}
$$

and

$$
\Delta T=\frac{\left[t_{1} \frac{\bar{W} \cdot(\bar{W}+2 \xi)}{\bar{W}+\xi}-t_{2} \cdot \bar{W}\right]\left(\frac{16 h \delta_{m} \delta_{n}}{3 \alpha \beta a b}\right)+\left(t_{3}+t_{30}\right) h^{2} \cdot \bar{W} \cdot(\bar{W}+2 \xi)-t_{4} \frac{\bar{W}}{\bar{W}+\xi}+\left(t_{2}-t_{20}-t_{21}\right) \bar{W}\left(\frac{4 h \delta_{m} \delta_{n}}{\alpha \beta a b}\right)}{\frac{\alpha^{2}+\beta^{2}}{1-v} H+\frac{\alpha^{2} E_{s x} \alpha_{1} b_{1}}{d_{1}} H_{x}+\frac{\beta^{2} E_{s y} \alpha_{2} b_{2}}{d_{2}} H_{y}},
$$

where

$$
\begin{aligned}
& E_{s x}=E_{s y}=E_{m}, \alpha_{1}=\alpha_{2}=\alpha_{m}, \\
& H=\frac{\sum_{p=0}^{\infty} \frac{1}{k p+1}\left(\frac{-K_{c m}}{K_{m}}\right)^{p} h\left(\frac{E_{m} \alpha_{m}}{k p+2}+\frac{E_{m} \alpha_{c m}+E_{c m} \alpha_{m}}{k p+k+2}+\frac{E_{c m} \alpha_{c m}}{k p+2 k+2}\right)}{\sum_{p=0}^{\infty} \frac{1}{k p+1}\left(\frac{-K_{c m}}{K_{m}}\right)^{p}}, \\
& H_{x}=\frac{\sum_{p=0}^{\infty} \frac{1}{k p+1}\left(\frac{-K_{c m}}{K_{m}}\right)^{p} h\left(\frac{-1}{k p+2}\right)\left(\frac{-h_{1}}{h}\right)^{k p+2}}{\sum_{p=0}^{\infty} \frac{1}{k p+1}\left(\frac{-K_{c m}}{K_{m}}\right)^{p}} \\
& H_{y}=\frac{\sum_{p=0}^{\infty} \frac{1}{k p+1}\left(\frac{-K_{c m}}{K_{m}}\right)^{p} h\left(\frac{-1}{k p+2}\right)\left(\frac{-h_{2}}{h}\right)^{k p+2}}{\sum_{p=0}^{\infty} \frac{1}{k p+1}\left(\frac{-K_{c m}}{K_{m}}\right)^{p}} .
\end{aligned}
$$

\subsubsection{Thermomechanical stability analysis}

Consider an imperfect ES-FGM plate simultaneously acted by a thermal field and an uniaxial compressive loading $P_{x}$, uniformly distributed along the edges $x=0$ and $x=a$. Suppose that the plate is simply supported with movable edges $x=0, a$ and immovable $y=0, b$ (Case (3)). Employing $N_{x 0}=-P_{x} h$ and the second of Eqs. (43), (44), 
we obtain

$$
\begin{aligned}
N_{y 0}= & \frac{-A_{12}^{*}}{A_{11}^{*}} P_{x} h-\frac{1}{A_{11}^{*}}\left[\left(\beta^{2} A_{12}^{*}-\alpha^{2} A_{11}^{*}\right) L_{3}+\alpha B_{21}^{*} L_{4}-\beta B_{22}^{*} L_{5}\right] W\left(\frac{4 \delta_{m} \delta_{n}}{\alpha \beta a b}\right) \\
& +\frac{1}{8 A_{11}^{*}} \beta^{2} W(W+2 \xi)-\left(1-\frac{A_{12}^{*}}{A_{11}^{*}}\right) \phi_{m}+\frac{A_{12}^{*}}{A_{11}^{*}} \phi_{m x}-\phi_{m y} .
\end{aligned}
$$

Substituting expressions of $N_{x 0}=-P_{x} h$ and $N_{y 0}$, taking into account the expressions of $\phi_{m}, \phi_{m x}, \phi_{m y}$ in (47), yields

$$
\begin{aligned}
P_{x}= & \frac{A_{11}^{*}}{h\left(\alpha^{2} A_{11}^{*}+\beta^{2} A_{12}^{*}\right)}\left\{\left[t_{1} \frac{\bar{W} \cdot(\bar{W}+2 \xi)}{\bar{W}+\xi}-t_{2} \cdot \bar{W}\right]\left(\frac{16 h \delta_{m} \delta_{n}}{3 \alpha \beta a b}\right)+t_{3} \cdot h^{2} \cdot \bar{W} \cdot(\bar{W}+2 \xi)\right. \\
& -t_{4} \frac{\bar{W}}{\bar{W}+\xi}-t_{5} \bar{W}\left(\frac{4 h \delta_{m} \delta_{n}}{\alpha \beta a b}\right)+\frac{\beta^{4}}{8 A_{11}^{*}} h^{2} \bar{W}(\bar{W}+2 \xi)+\left[\frac{\beta^{2} A_{12}^{*}}{A_{11}^{*}} \phi_{m x}^{0}\right. \\
& \left.\left.-\beta^{2} \phi_{m y}^{0}-\beta^{2}\left(1-\frac{A_{12}^{*}}{A_{11}^{*}}\right) \frac{h P}{1-v}\right] \Delta T-q \frac{1}{\bar{W}+\xi}\left(\frac{16 \delta_{m} \delta_{n}}{\alpha \beta a b h}\right)\right\},
\end{aligned}
$$

where

$$
t_{5}=\frac{\beta^{2}}{A_{11}^{*}}\left[\left(\beta^{2} A_{12}^{*}-\alpha^{2} A_{11}^{*}\right) L_{3}+\alpha B_{21}^{*} L_{4}+\beta B_{22}^{*} L_{5}\right] .
$$

Eq. (57) is employed to trace postbuckling load-deflection curves of the imperfect ES-FGM plates subjected to the combined mechanical and thermal loads. Besides, it is used to determine the dependence of the in-plane compressive edge loads vs. total deflection (for given uniform temperature rise).

\section{CONCLUSIONS}

This paper investigates the nonlinear post-buckling analysis of imperfect FGM plates reinforced by FGM stiffeners on elastic foundations subjected to in-plane compressive mechanical loads or thermal loads, or thermo-mechanical loads simultaneously by analytical approach. The material properties of plate and stiffeners are graded in the thickness direction according to a volume fraction power-law distribution. Based on the first order shear deformation theory with the kinematic nonlinearity and taking into account shear deformation of stiffener, temperature and Pasternak elastic foundation, the couple set of four nonlinear stability equations for functionally graded plates are derived. By applying Galerkin's method, the closed-form expressions for determining the buckling load and post-buckling load-deflection curves are obtained. The relations (11) and (12) are most important relations found in this work in which the thermal elements in plate and stiffeners in equations of $N_{i j}$ and $M_{i j}$ are considered. The effects of temperature, stiffener, material properties, geometrical parameters and foundation parameters will be analyzed in detail in the next part of the paper.

\section{ACKNOWLEDGEMENT}

This research is funded by the VNU University of Science under project number TN.15.03. 


\section{REFERENCES}

[1] S. P. Timoshenko and J. M. Gere. Theory of elastic stability. Mc Graw-Hill, New York, (1961).

[2] E. Steen. Elastic buckling and postbuckling of eccentrically stiffened plates. International Journal of Solids and Structures, 25, (7), (1989), pp. 751-768.

[3] O. K. Bedair. Influence of stiffener location on the stability of stiffened plates under compression and in-plane bending. International Journal of Mechanical Sciences, 39, (1), (1997), pp. 3349.

[4] J. N. Reddy. Analysis of functionally graded plates. International Journal for Numerical Methods in Engineering, 47, (1-3), (2000), pp. 663-684.

[5] W. Lanhe. Thermal buckling of a simply supported moderately thick rectangular FGM plate. Composite Structures, 64, (2), (2004), pp. 211-218.

[6] H. V. Tung and N. D. Duc. Nonlinear analysis of stability for functionally graded plates under mechanical and thermal loads. Composite Structures, 92, (5), (2010), pp. 1184-1191.

[7] A. Lal, K. R. Jagtap, and B. N. Singh. Post buckling response of functionally graded materials plate subjected to mechanical and thermal loadings with random material properties. Applied Mathematical Modelling, 37, (5), (2013), pp. 2900-2920.

[8] N. D. Duc and H. V. Tung. Mechanical and thermal postbuckling of higher order shear deformable functionally graded plates on elastic foundations. Composite Structures, 93, (11), (2011), pp. 2874-2881.

[9] Y. Kiani and M. R. Eslami. An exact solution for thermal buckling of annular FGM plates on an elastic medium. Composites Part B: Engineering, 45, (1), (2013), pp. 101-110.

[10] A. Naderi and A. R. Saidi. Exact solution for stability analysis of moderately thick functionally graded sector plates on elastic foundation. Composite Structures, 93, (2), (2011), pp. 629638 .

[11] M. M. Najafizadeh, A. Hasani, and P. Khazaeinejad. Mechanical stability of functionally graded stiffened cylindrical shells. Applied Mathematical Modelling, 33, (2), (2009), pp. 11511157.

[12] D. H. Bich, D. V. Dung, and V. H. Nam. Nonlinear dynamical analysis of eccentrically stiffened functionally graded cylindrical panels. Composite Structures, 94, (8), (2012), pp. 24652473.

[13] D. H. Bich, D. V. Dung, and V. H. Nam. Nonlinear dynamic analysis of eccentrically stiffened imperfect functionally graded doubly curved thin shallow shells. Composite Structures, 96, (2013), pp. 384-395.

[14] D. V. Dung and L. K. Hoa. Nonlinear buckling and post-buckling analysis of eccentrically stiffened functionally graded circular cylindrical shells under external pressure. Thin-Walled Structures, 63, (2013), pp. 117-124.

[15] D. V. Dung and L. K. Hoa. Research on nonlinear torsional buckling and post-buckling of eccentrically stiffened functionally graded thin circular cylindrical shells. Composites Part B: Engineering, 51, (2013), pp. 300-309.

[16] D. V. Dung and V. H. Nam. Nonlinear dynamic analysis of eccentrically stiffened functionally graded circular cylindrical thin shells under external pressure and surrounded by an elastic medium. European Journal of Mechanics-A/Solids, 46, (2014), pp. 42-53.

[17] D. V. Dung, L. K. Hoa, and N. T. Nga. On the stability of functionally graded truncated conical shells reinforced by functionally graded stiffeners and surrounded by an elastic medium. Composite Structures, 108, (2014), pp. 77-90. 
[18] N. D. Duc and P. H. Cong. Nonlinear postbuckling of an eccentrically stiffened thin FGM plate resting on elastic foundations in thermal environments. Thin-Walled Structures, 75, (2014), pp. 103-112.

[19] N. D. Duc and T. Q. Quan. Nonlinear postbuckling of imperfect eccentrically stiffened PFGM double curved thin shallow shells on elastic foundations in thermal environments. Composite Structures, 106, (2013), pp. 590-600.

[20] D. V. Dung and L. K. Hoa. Nonlinear torsional buckling and postbuckling of eccentrically stiffened FGM cylindrical shells in thermal environment. Composites Part B: Engineering, 69, (2015), pp. 378-388.

[21] L. X. Peng, S. Kitipornchai, and K. M. Liew. Analysis of rectangular stiffened plates under uniform lateral load based on FSDT and element-free Galerkin method. International Journal of Mechanical Sciences, 47, (2), (2005), pp. 251-276.

[22] J. N. Reddy. Mechanics of laminated composite plates and shells: Theory and analysis. CRC press, (2004).

[23] H. S. Shen. Functionally graded materials: Nonlinear analysis of plates and shells. CRC press, (2009).

[24] H. S. Shen. Thermal postbuckling behavior of shear deformable FGM plates with temperature-dependent properties. International Journal of Mechanical Sciences, 49, (4), (2007), pp. $466-478$.

[25] L. Librescu and M. Stein. A geometrically nonlinear theory of transversely isotropic laminated composite plates and its use in the post-buckling analysis. Thin-Walled Structures, 11, (1), (1991), pp. 177-201. 


\section{APPENDIX I}

$$
\begin{aligned}
& A_{11}=A_{22}=\frac{E_{1}}{1-v^{2}}, \quad A_{12}=\frac{E_{1} v}{1-v^{2}}, \quad A_{66}=\frac{E_{1}}{2(1+v)}, \\
& A_{44}=\chi_{1}\left[\frac{E_{1}}{2(1+v)}+\frac{G_{1 s} b_{1}}{d_{1}}\right], \quad A_{55}=\chi_{2}\left[\frac{E_{1}}{2(1+v)}+\frac{G_{1 r} b_{2}}{d_{2}}\right], \quad \chi_{1}=\chi_{2}=5 / 6, \\
& B_{11}=B_{22}=\frac{E_{2}}{1-v^{2}}, \quad B_{12}=\frac{E_{2} v}{1-v^{2}}, \quad B_{12}=\frac{E_{2} v}{1-v^{2}}, \quad B_{66}=\frac{E_{2}}{2(1+v)}, \\
& D_{11}=D_{22}=\frac{E_{3}}{1-v^{2}}, \quad D_{12}=\frac{E_{3} v}{1-v^{2}}, \quad D_{66}=\frac{E_{3}}{2(1+v)}, \\
& E_{1}=\left(E_{m}+\frac{E_{c}-E_{m}}{k+1}\right) h, \quad E_{2}=\frac{\left(E_{c}-E_{m}\right) k h^{2}}{2(k+1)(k+2)}, \\
& E_{3}=\left[\frac{E_{m}}{12}+\left(E_{c}-E_{m}\right)\left(\frac{1}{k+3}-\frac{1}{k+2}+\frac{1}{4 k+4}\right)\right] h^{3}, \\
& E_{1 s}=E_{m} h_{1}+E_{c m} \frac{h_{1}}{k_{2}+1}, \\
& E_{2 s}=-E_{m} \frac{h_{1}\left(h+h_{1}\right)}{2}-E_{c m}\left(\frac{h_{1}^{2}}{k_{2}+2}+\frac{h_{1} h}{2 k_{2}+2}\right), \\
& E_{3 s}=\frac{1}{12} E_{m}\left(3 h_{1} h^{2}+6 h_{1}^{2} h+4 h_{1}^{3}\right)+E_{c m}\left(\frac{h_{1}^{3}}{k_{2}+3}+\frac{h_{1}^{2} h}{k_{2}+2}+\frac{h_{1} h^{2}}{4 k_{2}+4}\right) \\
& E_{1 r}=E_{m} h_{2}+E_{c m} \frac{h_{2}}{k_{3}+1}, \\
& E_{2 r}=-E_{m} \frac{h_{2}\left(h+h_{2}\right)}{2}-E_{c m}\left(\frac{h_{2}^{2}}{k_{3}+2}+\frac{h_{2} h}{2 k_{3}+2}\right), \\
& E_{3 r}=\frac{1}{12} E_{m}\left(3 h_{2} h^{2}+6 h_{2}^{2} h+4 h_{2}^{3}\right)+E_{c m}\left(\frac{h_{2}^{3}}{k_{3}+3}+\frac{h_{2}^{2} h}{k_{3}+2}+\frac{h_{2} h^{2}}{4 k_{3}+4}\right) \\
& C_{1}=\frac{E_{2 s} b_{1}}{d_{1}}, \quad C_{2}=\frac{E_{2 r} b_{2}}{d_{2}}, \\
& b_{1}
\end{aligned}
$$

where $h_{1}, b_{1}$ and $h_{2}, b_{2}$ are the thickness and width of the longitudinal and transversal stiffeners, respectively; $d_{1}$ and $d_{2}$ are the distance between two longitudinal and transversal stiffeners, respectively,

$$
\begin{gathered}
\phi_{m}=\int_{-\frac{h}{2}}^{\frac{h}{2}} \frac{E(z)}{1-v} \alpha(z) \Delta T d z, \phi_{m x}=\frac{b_{1}}{d_{1}} \int_{-\frac{h}{2}-h_{1}}^{-\frac{h}{2}} E_{s x}(z) \alpha_{1}(z) \Delta T d z, \phi_{m y}=\frac{b_{2}}{d_{2}} \int_{-\frac{h}{2}-h_{2}}^{-\frac{h}{2}} E_{s y}(z) \alpha_{2}(z) \Delta T d z, \\
\phi_{b}=\int_{-\frac{h}{2}}^{\frac{h}{2}} \frac{z E(z)}{1-v} \alpha(z) \Delta T d z, \phi_{b x}=\frac{b_{1}}{d_{1}} \int_{-\frac{h}{2}-h_{1}}^{-\frac{h}{2}} z E_{s x}(z) \alpha_{1}(z) \Delta T d z, \phi_{b y}=\frac{b_{2}}{d_{2}} \int_{-\frac{h}{2}-h_{2}}^{-\frac{h}{2}} z E_{s y}(z) \alpha_{2}(z) \Delta T d z .
\end{gathered}
$$




\section{APPENDIX II}

$$
\begin{aligned}
& \Delta=\left(A_{11}+\frac{E_{1 s} b_{1}}{d_{1}}\right)\left(A_{22}+\frac{E_{1 r} b_{2}}{d_{2}}\right)-A_{12}^{2}, \quad A_{11}^{*}=\frac{1}{\Delta}\left(A_{11}+\frac{E_{1 s} b_{1}}{d_{1}}\right), \\
& A_{22}^{*}=\frac{1}{\Delta}\left(A_{22}+\frac{E_{1 r} b_{2}}{d_{2}}\right), \quad A_{12}^{*}=\frac{A_{12}}{\Delta}, \quad A_{66}^{*}=\frac{1}{A_{66}}, \quad B_{11}^{*}=A_{22}^{*}\left(B_{11}+C_{1}\right)-A_{12}^{*} B_{12}, \\
& B_{22}^{*}=A_{11}^{*}\left(B_{22}+C_{2}\right)-A_{12}^{*} B_{12}, \quad B_{12}^{*}=A_{22}^{*} B_{12}-A_{12}^{*}\left(B_{22}+C_{2}\right), \\
& B_{21}^{*}=A_{11}^{*} B_{12}-A_{12}^{*}\left(B_{11}+C_{1}\right), \quad B_{66}^{*}=\frac{B_{66}}{A_{66}}, \\
& D_{11}^{*}=D_{11}+\frac{E_{3 s} b_{1}}{d_{1}}-B_{11}^{*}\left(B_{11}+C_{1}\right)-B_{21}^{*} B_{12}, \\
& D_{22}^{*}=D_{22}+\frac{E_{3 r} b_{2}}{d_{2}}-B_{22}^{*}\left(B_{22}+C_{2}\right)-B_{12}^{*} B_{12}, \\
& D_{12}^{*}=D_{12}-B_{12}^{*}\left(B_{11}+C_{1}\right)-B_{22}^{*} B_{12}, \quad D_{21}^{*}=D_{12}-B_{21}^{*}\left(B_{22}+C_{2}\right)-B_{11}^{*} B_{12}, \\
& D_{66}^{*}=D_{66}-B_{66}^{*} B_{66} .
\end{aligned}
$$

\section{APPENDIX III}

$$
\begin{aligned}
& L_{1}=\frac{4 \alpha^{2} D_{11}^{*}+A_{44}}{A_{11}^{*}\left(4 \alpha^{2} D_{11}^{*}+A_{44}\right)+4 \alpha^{2} B_{21}^{* 2}} \cdot \frac{\beta^{2}}{32 \alpha^{2}}, \\
& 7 L_{2}=\frac{4 \beta^{2} D_{22}^{*}+A_{55}}{A_{22}^{*}\left(4 \beta^{2} D_{22}^{*}+A_{55}\right)+4 \beta^{2} B_{12}^{* 2}} \cdot \frac{\alpha^{2}}{32 \beta^{2}}, \\
& L_{3}=\frac{\left(A_{44} a_{22} \alpha-A_{55} a_{12} \beta\right) a_{13}+\left(A_{55} a_{11} \beta-A_{44} a_{21} \alpha\right) a_{23}}{D^{*}\left[\alpha^{4} A_{11}^{*}+\alpha^{2} \beta^{2}\left(A_{66}^{*}-2 A_{12}^{*}\right)+\beta^{4} A_{22}^{*}\right]+\left(a_{13} a_{22}-a_{12} a_{23}\right) a_{13}+\left(a_{11} a_{23}-a_{21} a_{13}\right) a_{23}}, \\
& L_{4}=\frac{L_{3}}{D^{*}}\left(a_{13} a_{22}-a_{12} a_{23}\right)-\frac{A_{44} a_{22} \alpha-A_{55} a_{12} \beta}{D^{*}}, \\
& L_{5}=\frac{L_{3}}{D^{*}}\left(a_{11} a_{23}-a_{21} a_{13}\right)-\frac{A_{55} a_{11} \beta-A_{44} a_{21} \alpha}{D^{*}}, \\
& L_{6}=\frac{8 \alpha^{3} B_{21}^{*}}{4 \alpha^{2} D_{11}^{*}+A_{44}} \cdot L_{1}, \quad L_{7}=\frac{8 \beta^{3} B_{12}^{*}}{4 \beta^{2} D_{22}^{*}+A_{55}} \cdot L_{2}, \quad D^{*}=a_{11} a_{22}-a_{12} a_{21}, \\
& a_{11}=\alpha^{2} D_{11}^{*}+\beta^{2} D_{66}^{*}+A_{44}, \quad a_{22}=\beta^{2} D_{22}^{*}+\alpha^{2} D_{66}^{*}+A_{55}, \quad a_{12}=\alpha \beta\left(D_{12}^{*}+D_{66}^{*}\right), \\
& a_{21}=\alpha \beta\left(D_{21}^{*}+D_{66}^{*}\right), \quad a_{13}=-\left[\alpha^{3} B_{21}^{*}+\alpha \beta^{2}\left(B_{11}^{*}-B_{66}^{*}\right)\right], \\
& a_{23}=-\left[\beta^{3} B_{12}^{*}+\alpha^{2} \beta\left(B_{22}^{*}-B_{66}^{*}\right)\right] .
\end{aligned}
$$

